ИЗВЕСТИЯ АКАДЕМИИ НАУК ЭСТОНСКОЙ ССР. ТОМ ХV СЕРИЯ ФИЗИКО-МАТЕМАТИЧЕСҚИХ И ТЕХНИЧЕСҚИХ НАУК. 1966, № 1

\author{
И. ТЕХВЕР, В. ХИЖНЯКОВ
}

\title{
КОМБИНАЦИОННОЕ РАССЕЯНИЕ И ЛЮМИНЕСЦЕНЦИЯ ВО ВТОРИЧНОМ СВЕЧЕНИИ ПРИМЕСНОГО ЦЕНТРА
}

\author{
Введение
}

Данное исследование является продолжением работ $[1,2]$, в которых была поставлена цель выяснить возможности комбинационного рассеяния света для исследовання примесных центров в кристаллах. Оно посвящено проблеме разделения вторичного свечения на комбинационное рассеяние и люминесценцию. Следует отметить, что теория резонансного комбинационного рассеяния, несмотря на наблюдающийся в последнее время заметный интерес к этой проблеме [3--5], развита недостатсчно. Наиболее псдробно. исследована модель с нулевой дисперсией (одно- или двухосцилляторная модель) в гармоническом приближении $\left[{ }^{3,5}\right]$. Однако, как следует из настоящей работы, эта моделі. недостаточна для описания рассеяния при возбуждении в области полосы поглощения. В теории резонансного комбинационного рассеяния необходимо учесть конечную ширину уровней спектра колебательных возбуждений $\Delta \varepsilon$ (в случае взаимодействия электронного перехода с одним локальным колебанием $\Delta \varepsilon=\Gamma$, где $\Gamma-$ ангармоническая константа затухания этого колебания), поскольку

$$
\Delta \varepsilon \gg \gamma
$$

$(\gamma-$ естественная ширина возбужденного электронного уровня). Иначе говоря, непєходимо учесть, что колебательная релаксация происходит гораздо быстрее (обычно іs: $10^{4}$ или больше раз) электронной релаксации. Спектр вторичного свечения при условии (1), как будет видно ниже, кардинальным образом отличается от спектра, получаемого при пренебрежении условием (1).* Благодаря учету (1) получим, что спектр вторичного свечения содержит интенсивную люминесценцию, получаемую обычно в первом порядке по взаимодействию света с электронами примеси, и гораздо более слабое, имеющее второй порядок по взаимодействию света с центром, комбинационное рассеяние.

\section{I. Комбинационное рассеяние и люминесценция во вторичном свечении:}

Используем такую же модель, как в работе [2]. Рассмотрим примесный кристалл с хаотическим распределением центров. Предполагаем, что для исследуемого процесса применимо адиабатическое приближение Борна-Оппенгеймера. Пусть на кристалл падает монохроматическая электромагнитная волна с напряженностью $\vec{E}(t)=\overrightarrow{E^{+}} e^{i \omega_{0} t}+\vec{E}-e^{-i \omega_{0} t}$, частота которой попадает в полосу поглощения примеси. В таком слу-

* Резкое изменение спектра вторичного свечения при учете условия (1) вполне аналогично исчезновению функций Бесселя нулевого порядка мнимого аргумента в формулах вероятности линии Мессбауэра и чистоэлектронной линии при наличии локальных колебаний [6, 7]. 
чае можем ограничиться приближением Кондона (учет отклонения от приближения Кондона тем более существенен, чем дальше рассеиваемая частота от полосы поглощения [ $\left.\left.{ }^{1}\right]\right)$.

Исходим из формулы, обычно используемой для вычисления интенсивности комбинационного рассеяния (см., напр., $\left.{ }^{8}\right]$ ). Учитывая вышеприведенные предположения, получим

$$
\begin{aligned}
W\left(\omega_{0}, \Omega\right)=B \omega_{0}^{4} \sum_{v_{s}, v_{s}^{\prime}} w\left(v_{s}\right)\left|\sum_{v_{s}^{\prime \prime}} \frac{\left\langle v_{s} \mid v_{s}^{\prime \prime}\right\rangle\left\langle v_{s}^{\prime \prime} \mid v_{s}^{\prime}\right\rangle}{\omega_{01}+\omega_{v_{s} v_{s}^{\prime \prime}}-\omega_{0}+i \gamma / 2}\right|^{2} \times \\
\times \delta\left(\Omega-\omega_{0}+\sum_{s} \omega_{v_{s} v_{s}^{\prime}}\right) .
\end{aligned}
$$

Здесь использованы следующие обозначения:

$$
\left.B=\frac{1}{2 \pi c^{3} n}|<0| \vec{E} \vec{M}|1\rangle<1|\vec{i} \vec{M}| 0\right\rangle\left.\right|^{2}
$$

$\vec{i}$ - единичный вектор поляризации рассеянного излучения, $\vec{M}-$ вектор дипольного момента электронов примесного центра; 0,1 - индексы основного и возбужденного электронных состояний соответственно, $v_{s}, v_{s}^{\prime}, v_{s}^{\prime}$ - числа заполнения начального, промежуточного и конечного колебательных состояний, w(vs) - фактор Больцмана.

Ниже будет показано, что формула (2) описывает не только рассеяние, но и люминесценцию. При этом подавляющая интенсивность всего вторичного свечения принадлежит люминесценции.

Для дальнейшего исследования формулы (2) представим ее в более конкретном виде, используя полученную в [2] формулу (8), учитывающую анггармонизм колебаний (изменением упругих постоянных при электронном переходе пренебрегаем)

$$
\begin{aligned}
& W\left(\omega_{0}, \Omega\right)=\frac{B \omega_{0}^{4}}{2 \pi} \int_{-\infty}^{\infty} d \mu \exp \left[i\left(\Omega-\omega_{0}\right) \mu\right] \int_{0}^{\infty} d t d t^{\prime} X \\
& \times \exp \left[i\left(\omega_{10}-\omega_{0}\right)\left(t^{\prime}-t\right)-\frac{\gamma}{2}\left(t^{\prime}+t\right)\right] A\left(t t^{\prime} \mu\right),
\end{aligned}
$$

где

$$
\begin{gathered}
A\left(t t^{\prime} \mu\right)=\exp \left[g(t)+g^{*}\left(t^{\prime}\right)+g^{*}(\mu)-g^{*}(\mu-t)-\right. \\
\left.-g^{*}\left(\mu+t^{\prime}\right)+g^{*}\left(\mu+t^{\prime}-t\right)\right] \\
g(t)=\sum_{s} \xi_{s}^{2}\left[\left(\bar{v}_{s}+1\right)\left(e^{-i \omega} s^{t-\Gamma_{s}|t|}-1\right)+\bar{v}_{s}\left(e^{i \omega} s^{t-\Gamma_{s}|t|}-1\right)\right]
\end{gathered}
$$

$\xi_{s}=x_{0 s} \sqrt{\omega_{s} / 2 \hbar}, x_{0 s}-$ сдвиг положения равновесия осциллятора $s$ при электронном переходе $0 \rightarrow 1$, $\omega_{s}$ - частота осциллятора, $\Gamma_{s}-$ константа ангармонического затухания осциллятора $s$.

Примем во внимание, что функция $\exp [g(t)]$ затухает с увеличением $|t|$, стремясь при $t \rightarrow \infty$ к пределу $\exp (-S)=\exp \left[-\sum_{s} \xi_{s}^{2}\left(2 \bar{v}_{s}+1\right)\right]$. Скорость затухания зависит от спектра частот колебаний, их ангармонических констант затухания и стоксовых потерь. Так, в случае сплош- 
ного колебательного спектра область значений $|t|$, в которой $\exp [g(t)]-$ - $\exp (-S)$ отлична от нуля, по порядку величины меньше или равна $1 / \bar{\omega}(\bar{\omega}-$ ширина фононного спектра; см. также раздел II настоящей работы). Если имеются локальные колебания, то $\exp [g(t)]-\exp (-S)$ заметно отличается от нуля в области $|t| \lesssim 1 / \Gamma_{\lambda}$ ( $\lambda$ нумерует локальные колебания). Итак, в предположении условия (1) область отличных от нуля значений функции $\exp [g(t)]-\exp (-S)$ гораздо меньше $1 / \gamma$. $W\left(\omega_{0}, \Omega\right)$.

Рассмотрим на основе указанных выше свойств $\exp [g(t)]$ спектр

В работах $\left[{ }^{2-5}\right]$ было показано, что при частоте $\Omega=\omega_{0}$ в спектре $W\left(\omega_{0}, \Omega\right)$ содержится $\delta$-образная особенность - релеевская линия рассеяния, вероятность которой $W_{0}$ определяется асимптотическим значением $A\left(t t^{\prime} \mu\right)$ при $\mu \rightarrow \infty$ :

$$
W_{0}=\left.\left.\frac{B \omega_{0}^{4}}{2 \pi}\right|_{0} ^{\infty} d t \exp \left[i\left(\omega_{10}-\omega_{0}\right) t-\frac{\gamma}{2} t+g^{*}(t)\right]\right|^{2} .
$$

Рассмотрим теперь $W\left(\omega_{0}, \Omega\right)$ для произвольных частот $\Omega$. При этом следует учитывать всевозможные значения $\mu$. Из указанных свойств $g(t)$ видно, что основной вклад в значение $W\left(\omega_{0}, \Omega\right)$ дает

$$
A^{(1)}\left(t t^{\prime} \mu\right)=\exp \left[g^{*}(\mu)+g^{*}\left(\mu+t^{\prime}-t\right)\right]
$$

отличное от нуля в области интегрирования $t+t^{\prime} \lesssim 1 / \gamma$. Действительно, при любом $\mu$ отброшенные члены отличны от нуля в области интегрирования $\left|t^{\prime}+\mu\right| \lesssim 1 / \Gamma_{\lambda} \ll 1 / \gamma,|t-\mu| \lesssim 1 / \Gamma_{\lambda} \ll 1 / \gamma$ (при отсутствии локальных колебаний $\Gamma_{\lambda}$ следует заменить на () ), т. е. дают вклад, по крайней мере, в $\Gamma_{\lambda} / \gamma$ раз меньший, чем $A^{(1)}\left(t_{t}^{\prime} \mu\right)$.

Подставим $A^{(1)}\left(t t^{\prime} \mu\right)$ в формулу (3). Введем переменные $t^{\prime}-t=x$, $t^{\prime}+t=y$, используем Фурье-преобразование

$$
e^{g^{*}(\mu+x)}=\int_{-\infty}^{\infty} F(z) e^{i(\mu+x) z} d z
$$

и проинтегрируем в формуле (3) по $x$ и $y$. Выполним затем обратное Фурье-преобразование и проинтегрируем по z. Получим

$$
\begin{aligned}
& W^{(1)}\left(\omega_{0}, \Omega\right)=\frac{B \omega_{0}^{4}}{2 \pi \nu} \int_{-\infty}^{\infty} d \mu \exp \left[i\left(\Omega-\omega_{10}\right) \mu+g^{*}(\mu)\right] \times \\
& \times \int_{-\infty}^{\infty} d \tau \exp \left[i\left(\omega_{10}-\omega_{0}\right) \tau+g^{*}(\tau)\right] \exp \left(-\frac{\gamma}{2}|\tau-\mu|\right) .
\end{aligned}
$$

В пренебрежении множителем $\exp \left(-\frac{\gamma}{2}|\tau-\mu|\right)$ формула (8) представляет собой произведение спектров поглощения

$$
\begin{aligned}
& I_{x}^{0}=\int_{-\infty}^{\infty} d \tau \exp \left[i\left(\omega_{10}-\omega_{0}\right) \tau+g^{*}(\tau)\right] \\
\text { и люминесценции } & I_{y}^{0}=\int_{-\infty}^{\infty} d \mu \exp \left[i\left(\Omega-\omega_{10}\right) \mu+g^{*}(\mu)\right],
\end{aligned}
$$

вычисленных без учета $\gamma$. Однако естественную ширину возбужденного 
электронного уровня можно не учитывать лишь в случае, когда $\omega_{0}$ и $\Omega$ попадают в области фононных крыльев полос поглощения и люминесценции.

а) Рассмотрим случай $\left|\omega_{0}-\omega_{10}\right| \lesssim \gamma$ ( $\omega_{0}$ находится в резонансе с чистоэлектронной линией). Тогда можно пренебречь $g^{*}(\tau)$ и формула (8) приводится к виду

$$
\begin{aligned}
& W^{(1)}\left(\omega_{0}, \Omega\right)=\frac{B \omega_{0}^{4}}{2 \pi} \cdot \frac{\exp \left[-\sum_{s} \xi_{s}^{2}\left(2 \overline{v_{s}}+1\right)\right]}{\left(\omega_{10}-\omega_{0}\right)^{2}+\gamma^{2} / 4} \times \\
& \times \int_{-\infty}^{\infty} d \mu \exp \left[i\left(\Omega-\omega_{0}\right) \mu+g^{*}(\mu)\right] .
\end{aligned}
$$

Из этой формулы следует, что при $\omega_{0} \approx \omega_{10}$ спектр близок к обычному спектру люминесценции примесного центра. Он отличается от последнего тем, что вместо чистоэлектронной линии радиационной ширины стоит $\delta$-образная релеевская линия $\Omega=\omega_{0}$, сдвинутая относительно чистоэлектронной линии на величину $\omega_{0}-\omega_{10}$. Этот результат, являющийся обобщением аналогичного результата для резонансного рассеяния атомами [9], был получен ранее одним из авторов в [2].

б) Если $\left|\omega_{0}-\omega_{10}\right| \gg \gamma$, то в области значений $\mu \gg 1 / \Gamma$, в которой нужно учитывать $\gamma$, можно $\gamma|\tau-\mu|$ заменить на $\gamma|\mu|$. Это дает

$$
W^{(1)}\left(\omega_{0}, \Omega\right)=\frac{B \omega_{0}^{4}}{2 \pi \nu} I_{y}(\Omega) I_{x}^{0}\left(\omega_{0}\right) .
$$

Мы видим, что спектр, описываемый формулой (2), при возбуждении в полосе поглощения действительно содержит люминесценцию (в которой имеется чистоэлектронная линия радиационной ширины $\gamma$ ). Полученный результат является вполне естественным, так как формула (2) включает в себя все вторичное свечение. При этом выделение люминесценции оказывается возможным только в случае, когда возбуждающий свет попадает в область фононного крыла полосы поглощения.

Напоминаем еще раз, что разделение спектров возможно из-за естественннгг условия (1). Как известно, в теории люминесценции $\left[{ }^{10}\right]$ исходным положением является то, что излучение происходит из возбужденного электронного состояния с равновесным распределением по колебательным степеням свободы. Обосновывается это тем, что в возбужденном электронном состоянии центр люминесценции находится долго и поэтому успеет установиться тепловое равновесие по колебаниям. Очевидно, что условие (1) $ү \ll \Gamma_{\lambda}(\bar{\omega})$ как раз выражает этот факт.

Неучтенные при получении формул (8) члены имеют интенсивность, по крайней мере, в $\Gamma / \gamma \sim 10^{4}$ раз меньше. Это не означает, что ими можно полностью пренебречь. Действительно, среди этих членов содержатся поправки к форме спектра люминесценции, которые можно не учитывать. Однако остальные члены описывают релеевскую линию и ее колебательные повторения, т. е. спектр комбинационного рассеяния [2]. Этот спектр расположен в другой области по сравнению с люминесценцией. Поэтому он, несмотря на малую интенсивность, может наблюдаться. 
Следует подчеркнуть также, что полученный здесь результат представляет собой прямое доказательство постоянства квантового выхода люминесценции и независимости спектра люминесценции от частоты возбуждения в пределах полосы примесного поглощения.

\section{II. ПРИМЕРЫ}

Ниже в качестве примера рассмотрен спектр вторичного свечения в двух конкретных случаях: 1) одноосцилляторная модель; 2) центр с большими стоксовыми потерями.

\section{1. Спектр вторичного свечения в случае одноосцилляторной модели}

Рассмотрим спектр одноосцилляторной модели при $T=0$. В таком случае

$$
g(t)=\xi^{2}\left(e^{-i \omega t-\Gamma|t|}-1\right) .
$$

Разлагая в (3) $A\left(t t^{\prime} \mu\right)$ в ряд и проводя интегрирование, получим

$$
\begin{gathered}
W\left(\omega_{0}, \Omega\right)=\frac{B \omega_{0}^{4}}{2 \pi} e^{-2 \xi^{2}} \sum_{\substack{m, m^{\prime} \\
l, l^{\prime}=0 \\
p, k}}^{\infty}(-1)^{l+l^{\prime}} \frac{\xi^{2\left(m+m^{\prime}+l+l^{\prime}+p+k\right)}}{m ! m^{\prime} ! l ! l^{\prime} ! p ! k !} \times \\
\times\left\{\left[i\left(\Omega-\omega_{10}+\omega\left(p-m+l^{\prime}\right)\right)-\gamma / 2-\Gamma\left(p+m+l^{\prime}\right)\right]^{-1} \times\right. \\
\times\left[( i \omega ( m - m ^ { \prime } + l - l ^ { \prime } ) + \gamma + \Gamma ( m + m ^ { \prime } + l + l ^ { \prime } ) ) ^ { - 1 } \left(f\left(m^{\prime}+l^{\prime}+k\right)-\right.\right. \\
\left.--f\left(m^{\prime}+l^{\prime}-k\right)\right)+f\left(m^{\prime}+l^{\prime}+k\right) f^{*}(m-l-k)- \\
\left.-f\left(m^{\prime}+l^{\prime}-k\right) f^{*}(m+l+k)\right]-\left[i\left(\Omega-\omega_{0}+\omega\left(p+k+l+l^{\prime}\right)\right)-\right. \\
\left.\left.-\Gamma\left(p+k+l+l^{\prime}\right)\right]^{-1} f\left(m^{\prime}+l^{\prime}+k\right) f^{*}(m-l-k)+C c\right\},
\end{gathered}
$$

где $f\left(m^{\prime} \pm l^{\prime} \pm k\right) \equiv\left[i\left(\omega_{10}-\omega_{0}+\omega\left(m^{\prime}+l^{\prime}+k\right)\right)-\gamma / 2-\Gamma\left(m^{\prime} \pm l^{\prime} \pm k\right)\right]^{-1} ; \quad$ С с означает комплексно сопряженные члены.

Рассмотрим члены, соответствующие $m=m^{\prime}=l=l^{\prime}=0 \quad$ (т. е. $g(t)+g^{*}\left(t^{\prime}\right)-$ $\left.-g^{*}(\mu-t)-g^{*}\left(\mu+t^{\prime}\right)=0\right)$ в случае $\omega_{0} \neq \omega_{10}$. Основной вклад в (9) дают члены:*

$$
\begin{gathered}
W^{(1)}\left(\omega_{0}, \Omega\right)=\frac{B \omega_{0}^{4}}{2 \pi \nu} e^{-2 \xi^{2}} \sum_{p=0}^{\infty} \frac{\xi^{2 p}}{p !} \frac{1}{i\left(\Omega-\omega_{10}+\omega p\right)-\gamma / 2-\Gamma p} \times \\
X \sum_{k=1}^{\infty} \cdot \frac{\xi^{2 k}}{k !}\left[\frac{1}{i\left(\omega_{10}-\omega_{0}+\omega k\right)-\gamma / 2-\Gamma k}-\frac{1}{i\left(\omega_{10}-\omega_{0}+\omega k\right)-\gamma / 2+\Gamma k}\right]+C c \approx \\
\approx \frac{B \omega_{0}^{4}}{2 \pi \nu} e^{-2 \xi^{2}} \sum_{p=0}^{\infty} \frac{(\gamma+2 \Gamma p) \xi^{2 p} / p !}{\left(\Omega-\omega_{10}+\omega p\right)^{2}+(\gamma / 2+\Gamma p)^{2}} \sum_{k=1}^{\infty} \frac{2 \Gamma k \xi^{2 k} / k !}{\left(\omega_{10}-\omega_{0}+\omega k\right)^{2}+(\Gamma k)^{2}} .
\end{gathered}
$$

Сомножитель, содержащий сумму по $p$, дает люминесценцию, а сумма по $k$ соответствует спектру поглощения. Таким образом, спектр вторичного свечения действительно содержит люминесценцию.

* Все остальные члены меньше учтенных по крайней мере в $\Gamma / \gamma$ раз. 
Нетрудно видеть, что члены $m, m^{\prime}, l l^{\prime} \neq 0$ содержат релеевскую линию $\left(\Omega=\omega_{0}\right)$ и ее колебательные повторения $\Omega=\omega_{0}-\omega P$.

Рассмотрим для примера интенсивность этих линий, если частота возбуждающего света совпадает с максимумом одной из линий поглощения, т. е. $\omega_{10}-\left(\omega_{0}+M \omega=0\right.$ $\left(M=m+l+k=m^{\prime}+l^{\prime}+k\right)$. Тогда интенсивность линии номера $P<M \quad(P=$ $=p+k+l+l^{\prime} \neq 0$ ) дается формулой

$$
\begin{gathered}
W_{P}=\frac{B \omega_{0}^{4}}{\pi \Gamma^{3} P M} e^{-2 \xi^{2}} \sum_{k=0}^{P} \sum_{l=0}^{P-k} \sum_{l^{\prime}=0}^{P-k-l}(-1)^{l+l^{\prime}} \times \\
X \frac{\xi^{2\left[P-l-l^{\prime}+2(M-k)\right]}(P+M-k)}{k ! l ! l^{\prime} !(M-l-k) !\left(M-l^{\prime}-k\right) !\left(P-l-l^{\prime}-k\right) !(M-k)(P+M-2 l-2 k)}
\end{gathered}
$$

Сравнивая формулы (10) и (11), убеждаемся, что комбинационное рассеяние дей ствительно по порядку величины в $\Gamma / \gamma$ раз слабее люминесценции.

Если полоса поглощения состоит из совершенно неперекрывающихся квазилиний $(\omega \gg \Gamma)$ и возбуждающий свет попадает между линиями поглощения, то $I_{x}\left(\omega_{0}\right)$ в. (8б), (10) практически равно нулю. Нетрудно заметить, что тогда $\Gamma$ можно пренебречь. Это дает результаты Е. Трифонова и К. Пойкера [5]. Таким образом, используемая в [5] модель применима лишь в этом специальном случае.

\section{2. Спектр вторичного свечения для центра с большими стоксовыми тотерями}

Для экспериментального разделения спектров люминесцечции и комбинационного рассеяния во вторичном свечении при возбуждении. в полосе поглсщения примеси особенно благоприятным является случай больших стоксовых потерь

$$
\sum_{s} \xi_{s}^{2}\left(2 \bar{v}_{s}+1\right) \gg 1
$$

Условие (12) позволяет разлагать функции $g(t), g^{*}\left(t^{\prime}\right)$ в ряд по $t$ и $t^{\prime}\left[{ }^{2}\right]$, ограничиваясь членами до второй степени включительно

$$
\exp [g(t)]-\exp (-S) \approx \exp \left[-\sum_{s} \xi_{s}^{2}\left(i \omega_{s} t+\frac{2 \bar{v}_{s}+\cdot 1}{2} \omega_{s}^{2} t^{2}\right)\right]
$$

(здесь учтено, что $\Gamma_{s} \ll \omega_{s}$ ). Как видно, функция (13) под интегралом по $t$ заметно отличается от нуля только в области

$$
t \lesssim \frac{1}{\sqrt{S_{2}}} \ll \frac{1}{\gamma}, \quad S_{2}=\sum_{s} \xi_{s}^{2} \omega_{s}^{2} \frac{\bar{v}_{s}+1}{2}
$$

что обеспечивает отделение спектра комбинационного рассеяния от спектра люминесценции. Интенсивность последней определяется выражением (3) в пренебрежении членами $\exp \left[g(t)+g^{*}\left(t^{\prime}\right)-g^{*}(\mu-t)-g^{*}\left(\mu+t^{\prime}\right)\right]$. Принимая во внимание (13) іл интегрируя по $t, t^{\prime}$ и $\mu$, получим формулу (8б), в которой для рассматриваемой модели, как хорошо известно, $I_{y}(\Omega)$ и $I_{x}\left(\omega_{0}\right)$ представляют собой суммы широких гауссовых кривых и чистоэлектронной линии ширины $\gamma$. Учет оставшихся членов дает релеевскую линию и ее колебательные повторения (комбинационное рассеяние), исследованные для данного случая в работе [2], а также поправки второго порядка к люминесценции. *

*. В работе $\left.{ }^{2}\right]$ не были учтены члены типа

$$
e^{-S} e^{-g^{*}(\mu-t)-g^{*}\left(\mu+t^{\prime}\right)}\left[e^{g(t)}+e^{g^{*}\left(t^{\prime}\right)}+e^{-S}\right] .
$$

Они дают несущественные поправки как к спектру люминесценции $\left(\sim \mathcal{V} / \sqrt{S_{2}}\right)$, так и к спектру комбинационного рассеяния $(\sim \exp (-S))$. 
Исследование спектра комбинационного рассеяния, проведенное в [2], показало, что в случае больших стоксовых потерь в спектре может наблюдаться квазилинейчатая структура (в отличие от спектра люминесценции, сплошного в случае болыших стоксовых потерь). Благодаря последнему обстоятельству условия для наблюдения колебательной структуры в комбинационном рассеянии более благоприятны. Это говорит о перспективности использования резонансного комбинационного рассеяния для изучения примесных центров поглощения с большими стоксовыми потерями.

Авторы приносят искреннюю благодарность К. К. Ребане за руководство настоящей работой.

\section{ЛИ Т Е Р А Т У P A}

1. Ребане К. К., Техвер И. Ю., Тр. Ин-та физ. и астрон. АН ЭССР, № 29, 54 (1964).

2. Техвер И. Ю., Тр. Ин-та физ. и астрон. АН ЭССР, № 32 (в печати).

3. П ерлин Ю. Е., ФТТ, 2, 1915 (1960); Перлин Ю. Е., Розенфельд Ю. Б., Уч. зап. Қишиневского гос. ун-та, 75, 1 (1964).

4. Дзюб И. П., Лубченко А. Ф., ФТТ, 3, 3602 (1961); Лубченко А. Ф., П а в лик Б. М., ФТТ, 5, 1714 (1963).

5. Три фонов Е. Д., Пойкер К., ФТТ, 7, 2345 (1965).

6. Кри воглаз М. А., Ж. эксперим. и теор. физ., 46, 637 (1964).

7. Хи жняков В. В., Изв. АН ЭССР. Сер. физ.-матем. и техн. наук, 14, № 1, 94 (1965).

8. Борн М. и Хуан Кунь, Динамическая теория кристаллических решеток, М., 1958.

9. Г ай т л е р В., Квантовая теория излучения, М., 1965.

10. Пек а р С. И., Ж. эксперим. н теор. физ., 22, 641 (1952).

Ннститут физики и астрономии Академии наук Эстонской ССР
Поступила в редакцию. $19 / \times 1965$

\section{TEHVER, V. HIZ̈NJAKOV}

\section{KOMBINATSIOONHAJUMINE JA LUMINESTSENTS LISANDITSENTRI SEKUNDAARSES KIIRGUSES}

Artiklis käsitletakse resonantskombinatsioonhajumise üht põhiprobleemi, nimelt: kuidas eraldada sekundaarse kiirguse spektris, mis on saadud ergutamisel lisandi neeldumisalas, kombinatsioonhajumine luminestsentsist. On näidatud, et tavaline kombinatsioonhajumise intensiivsuse arvutamiseks kasutatav valem sisaldab ka luminestsentsi. Viimaseväljaeraldamiseks on arvestatud tingimust $\Gamma_{S} \gg \gamma$ (s. t. kristalli võnkumiste relaksatsiooni: aeg on palju väiksem ergutatud elektronseisundi loomulikust elueast). On uuritud lisanditsentri sekundaarse kiirguse spektrit kahel juhul: a) kui ergutava valguse sagedus $\omega_{0}$ kuulub puhtelektronüleminekualasse; b) kui $\omega_{0}$ langeb neeldumisala foonontiiba. Esimesel juhul saadud spekter meenutab oma kujult luminestsentsispektrit, kuid puhtelektronjoont asendab Rayleigh' joon. Teisel juhul on võimalik kahte nimetatud spektrit eraldada. Näitena on vaadatud ühe ostsillaatori mudeli ja suurte Stokes'i kadudega lisanditsentri sekundaarse kiirguse spektrit. Teine näide väärib erilist tähelepanu, sest siin on terava kvasijoon-struktuuriga kombinatsioonhajumise spekter resonantsalas hästi eraldatav. Et luminestsentsispekter antud juhul kujutab endast pidevat struktuurita riba, siis just kombinatsioonhajumise spekter võib anda informatsiooni selliste lisanditsentrite kohta. 


\section{TEHVER, V. HIZHNYAKOV}

\section{RAMAN EFFECT AND LUMINESCENCE IN SECONDARY RADIATION BY AN IMPURITY CENTRE}

An essential problem of the theory of resonance Raman spectra, the separating of luminescence in the spectrum of secondary radiation, is discussed. Observing the process in adiabatic and Condon approximations, it is shown that the initial formula ordinarily used for the calculation of the intensity of the resonance Raman effect includes luminescence as well. In order to separate the Raman and luminescence spectra, the condition that $\Gamma_{s} \gg \gamma$ (i. e. the time of the relaxation of normal modes being much less than the radiative life-time of the excited electronic level) is taken into account. The spectrum of the secondary radiation of an impurity centre has been studied in two cases: a) the incident light $\omega_{0}$ being in the region of pure-electronic transition $\left(\left|\omega_{0}-\omega_{10}\right| \lesssim \gamma\right)$ and b) $\omega_{0}$ falling i1 to the region of the phonon wing. In the first case the spectrum is analogical in its form to the luminescence spectrum, but instead of a pure-electronic line there is a Rayleigh line shifted by value $\left|\omega_{0}-\omega_{10}\right| \lesssim \gamma$. In the second case the separation of Raman and luminescence spectra is possible. Two examples are discussed: the spectrum of the secondary radiation in the case of one-oscillator model and of an impurity centre with appreciable Stokes-shifts. In the latter case the quasilinear structure of a resonance Raman spectrum may be observed (while the luminescence spectra of such centres have continuous bands) - the circumstances allowing to use the resonance Raman spectra for studying the dynamics of impurity centres with appreciable Stokes-shifts. 